\title{
Endoscopic Surgery for Benign and Malignant Sinonasal Neoplasms
}

\author{
Raymond Sacks ${ }^{1,2,3}$ \\ ${ }^{1}$ Professor and Head Dept ORL/ Head \& Neck Surgery-Macquarie University, Australia \\ ${ }^{2}$ President Australasian Rhinologic Society \\ ${ }^{3}$ Chief Examiner in Otolaryngology/Head \& Neck Surgery- Royal Australasian College of Surgeons
}

Sinonasal neoplasms were traditionally treated by open approaches. In the past decade, endoscopic approaches have become more widely utilised and are proving to be as effective if not superior in tumour control. Benign sinonasal tumours encompass a collection of pathologies including fibroosseous lesions and neoplastic masses. Malignant tumours are relatively rare and account for only $1 \%$ of all malignancies. The annual incidence for malignant sinonasal tumours is about $0.5-1$ new case per 100,000 inhabitants. This is said to be higher in areas such as Asia and Africa where the incidence is between 2.5 and 2.6 per 100,000 per annum in Japan. Sinonasal malignancies are more common in males with a male to female ratio reported to be about 2:1. The most common sinonasal malignancy are the primary endothelial tumours followed by the non epithelial malignant tumours.

Complete tumour removal is always the desired end point with malignancy; total en bloc excision needs to be achieved with clear margins. Significant normal tissue removal may however be excessive in benign neoplasia and unacceptable cosmesis, functional impairment, dental disruption, epiphora, orbital displacement and nerve injury are acceptable outcomes for malignant tumours but not for benign and therefore there needs to be significant consideration of morbidity. The areas of high morbidity include the dura, the periorbita and small breaches may lead to intracranial and intraorbital recurrence and therefore require more radical surgery with more significant morbidity. The aids of technology such as coblation have dramatically improved our ability to clear malignant and benign neoplasia from the sinonasal cavity and this has led to a significant improvement in mortality and morbidity rates with such treatment. The development of vascularised flaps for effective and reliable reconstruction of the endoscopic skull base has also led to a significant improvement in our ability to resect these tumours. This talk will display the techniques utilized for such reconstruction and show examples of multiple different tumour approaches. The extent of endoscopic ability to reliably resect such tumours will be explained and demonstrated.

\section{Image Guidance in Modern Day Rhinology and the Evidence for It}

This talk will focus on navigation using computerised stereotactic imaging and will discuss the issues with image guided surgery. My experience over the past 15 years with image guidance will be highlighted and a comparison of both electromagnetic and optokinetic systems will be made. Examples of usage of image guidance will be highlighted and the limitations of image guidance highlighted as well. Finally, the question of evidence based image guidance usage will be answered with a meta-analysis and systematic review of all papers comparing the use of image guided and nonimage guided surgery. The complication rates and relative risks associated with image guidance will be evaluated and presented and both orbital and intracranial complications discussed. The conclusion of our meta-analysis is the formation of Level 2A evidence that image guidance surgery is associated with a lower risk of both major and total complications compared to non-image guided sinus surgery in selected populations and finally the recommendation for intraoperative image guidance surgery will be highlighted and presented. 